\title{
Soft X-Ray Diffraction up to Wavelengths of $6.0 \AA$ Becomes Feasible at ID1 of ESRF
}

\author{
P. Carpentier ${ }^{a}$, P. Boesecke ${ }^{b}$, J.-M. Bois ${ }^{c}$, M.-L. Chesne $^{a}$, \\ E. FANCHON ${ }^{a}$, R. $\mathrm{KAHN}^{a}, \mathrm{H} . \mathrm{STUHRMANN}^{a, d, *}$ AND J. $\operatorname{VICAT}^{a}$ \\ ${ }^{a}$ Institut de Biologie Structurale Jean-Pierre Ebel (IBS), CEA/CNRS \\ 41 rue Jules Horowitz, 38027 Grenoble, France \\ ${ }^{b}$ European Synchrotron Radiation Facility (ESRF) \\ Grenoble, France \\ ${ }^{c}$ European Molecular Biology Laboratory (EMBL) \\ Grenoble, France \\ ${ }^{d}$ GKSS Forschungszentrum, Geesthacht, Germany
}

\begin{abstract}
Recent experiments carried out at the Anomalous Scattering Beamline ID1 of ESRF are described which show that a cold helium atmosphere provides optimal conditions for protein crystallography with soft X-rays at wavelengths up to $6 \AA$. Image plates are suitable detectors for this wavelength range. The improvement with respect to earlier experiments is considerable. This observation opens the way to the use of anomalous dispersion at the $K$-edge of light elements, like phosphorus, sulphur, chlorine and calcium. Moreover, there is an interest to use the strong anomalous dispersion of some heavy elements such as uranium (110 anomalous electron units at its $M_{\mathrm{V}}$ edge) to solve large biological structures. The methods of multiwavelength anomalous diffraction and diffraction anomalous fine structure find new resonant labels which are wide spread in living matter and materials.
\end{abstract}

PACS numbers: $61.10 . \mathrm{Nz}$

\section{Introduction}

The use of soft X-ray diffraction in protein crystallography and in materials science is triggered by the access to larger signals of anomalous diffraction. There are light atoms like sulphur and phosphorus which are wide spread in living

* corresponding author: H.B. Stuhrmann, Institut de Biologie Structurale Jean-Pierre Ebel, CEA/CNRS, 41 rue Jules Horowitz, 38027 Grenoble, France 
organisms, minerals, fossil fuels, and in many kinds of materials. The imaginary increment $f^{\prime \prime}$ of resonant scattering from these elements increases with the square of the wavelength and culminates at the $K$ absorption edges between 5 and $6 \AA$ (Table). Moreover, there are some very heavy atoms like uranium and thorium with

TABLE

The X-ray penetration depth calculated for absorption edges of selected elements.

\begin{tabular}{l|l|c|c|c}
\hline \hline \multicolumn{1}{c|}{ Element } & Edge & Wavelength $[\AA]$ & Energy $[\mathrm{eV}]$ & $\begin{array}{c}\text { Penetration depth } \\
\text { in water }[\mu \mathrm{m}]\end{array}$ \\
\hline phosphorus & $K$ & 5.784 & 2143 & 19 \\
sulphur & $K$ & 5.018 & 2470 & 29 \\
chlorine & $K$ & 4.397 & 2820 & 43 \\
uranium & $M_{\mathrm{V}}$ & 3.497 & 3545 & 85 \\
uranium & $M_{\mathrm{IV}}$ & 3.333 & 3720 & 100 \\
calcium & $K$ & 3.070 & 4038 & 125
\end{tabular}

$M$ absorption edges between 3 and $4 \AA$, which are not only strong scatterers by themselves but also sources of an even stronger anomalous dispersion. In spite of the brilliant perspectives of anomalous soft X-ray diffraction in structural studies, it is only since rather recently when the first steps towards the use of wavelengths significantly beyond $1.5 \AA$ were undertaken. The main obstacle is the strong increase in absorption with longer wavelength. Moreover, the diffraction pattern at a given structural resolution will extend to larger angles, and at very large angles (close to backward diffraction) one encounters the structural resolution limit which is given by half of the X-ray wavelength. With wavelengths close to the $K$-absorption edge of sulphur, $\lambda_{k}=5.018 \AA$ the achievable structural resolution will stay below $2.5 \AA$. A fairly unconventional detector would be needed to allow for simultaneous measurement of the diffraction peaks over a solid angle which covers a reasonably large fraction $(>0.5)$ of the unit sphere. As a consequence, existing software packages need to be adapted to a new kind of diffraction geometry. Moreover, a closer view has to be given to absorption corrections and scaling procedures.

Two main lines of using soft $\mathrm{X}$-ray diffraction are presently followed:

(1) The off-edge strategy uses wavelengths between 1.5 and $3.0 \AA[1,2]$. It profits from the increase in the imaginary part $f^{\prime \prime}$ of anomalous dispersion, which for light elements in this wavelength range increases with the square of the wavelength for a moderate increase in X-ray absorption. With the maximum scattering angle of $2 \theta=60^{\circ}$ the resolution limit is equal to the wavelength. The choice of the wavelength is the result of bargaining between highest possible resolution, lowest absorption, and largest anomalous signal. 
The method uses the anomalous scattering at a single wavelength (single wavelength anomalous diffraction, SAD) [3]. An inherent disadvantage of SAD is that it is impossible to obtain a unimodal phase probability distribution. In order to resolve the resulting phase ambiguity it is therefore necessary to combine the SAD approach with density modification or other techniques. Moreover, the method is not specific to a single element. Typically, phosphorus, sulphur, chlorine, potassium and calcium will contribute to SAD in a comparable way $[4,5]$.

No major modifications of existing X-ray diffractometers at synchrotron radiation beamlines are required. Standard software packages for the analysis of the diffraction data in combination with local or detector scaling procedures are used successfully. This technique may have the potential to become one of the standard approaches to nearly completely automated protein structure determination [5].

(2) The near-edge strategy is bound to wavelengths near the absorption edge of some element. With sulphur as anomalous scattering atom, X-ray diffraction experiments will have to be carried out at wavelengths near $5 \AA$. The method of choice is that of the multiple wavelength anomalous dispersion diffraction (MAD) [6]. Phase determination is unique. This method is not only specific to a given element but also, it may discriminate between different chemical states of one and the same element. Taking sulphur as an example, the dispersion of sulphur in the valence state -2 (sulphide, sulphur in amino acids methionine and cystein) is shifted by $10 \mathrm{eV}$ to lower energies with respect to sulphur in the state +6 (sulphates). The method of MAD treats the two species of sulphur as separate entities [7]. This is less evident for the distinction between cystein and cystin, where the disulphide bridge of cystin leads to a splitting of the main peak of the absorption edge into two peaks separated by $2 \mathrm{eV}$ only. At this point, the measurement of the diffraction anomalous fine structure (DAFS) would be the more promising way $[8,9]$. The wealth of chemical information might well outweigh the reduced structural resolution.

For light elements, like phosphorus, sulphur and chlorine, the $K$-absorption edges present the only way to use the method of MAD. The $K$ absorption edges of these elements are found at wavelengths between $4 \AA$ and $6 \AA$ where the penetration depth of X-rays in almost any kind of matter is considerably reduced (Table).

The penetration depth of soft X-rays in organic matter is of the order of some tens of microns. For air - these numbers count, to a good approximation, in millimetres. Hence, a few $\mathrm{cm}$ of air reduces the intensity of soft X-rays at wavelengths cited above to about one third. The design of a beamline transparent for soft X-rays is a technical challenge. There is also a need for an area detector covering a large solid angle in order to reach the structural resolution limit of about $3 \AA$.

The consequences of increased absorption are less dramatic for the sample, as there is an increase in the scattering power with the square of the wavelength 


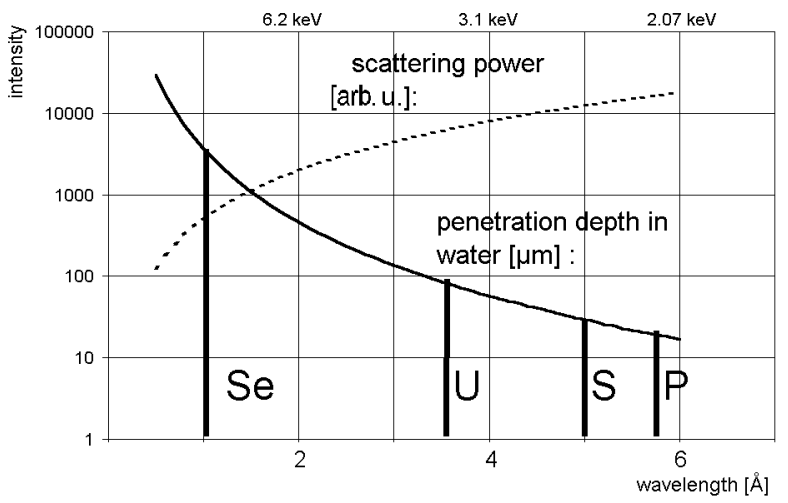

Fig. 1. Variation of the penetration depth of X-rays in water (in $\mu \mathrm{m}$ ) and the scattering power of a crystal (arbitrary units) versus the wavelength.

(Fig. 1). For very small crystals of micrometer size or for even smaller objects like single particles, the use of soft $\mathrm{X}$-rays may be a real advantage.

This paper deals with soft $\mathrm{X}$-ray diffraction at wavelengths beyond $3 \AA$. The approach to solve the technical problems encountered with the method of MAD as a near-edge method has been a stepwise one. Starting from the experience gained at the beam line A1 of HASYLAB [10,11], a series of test experiments of soft $\mathrm{X}$-ray diffraction have been carried out at beam line ID1 of ESRF [12, 13]. The present state will be discussed below.

\section{The experiment}

The experimental setup used for soft X-ray diffraction is an ancillary equipment designed for the implementation at the beam line ID1 of ESRF. It consists of a helium box which at its largest face carries an image plate with a sensitive area of $350 \mathrm{~mm} \times 430 \mathrm{~mm}$ (Fig. 2). A jet of cold helium ( $T=40 \mathrm{~K}$ ) is directed towards the protein crystal. The cryogenic facility, which has been developed at the EMBL Outstation at Grenoble, is an open stream system.

The sample was tetragonal lysozyme $(a=b=78.35 \AA, c=37.47 \AA)$. The crystals were obtained from a solution of $0.9 \mathrm{M} \mathrm{NaCl}, 50 \mathrm{mM} \mathrm{CH} \mathrm{CHOONa}_{3}$ at pH 4.5.

A crystal was mounted on a loop and washed with paraffin oil for 30 seconds, and immediately thereafter exposed to cold helium gas stream inside the helium box.

The X-ray optics of the beam line ID1 with its double silicon crystal monochromator delivers monochromatic radiation with wavelengths ranging from $0.3 \AA$ to $3 \AA$ and from $0.6 \AA$ to $6 \AA$ using the 311 and the 111 reflection of the silicon crystal, respectively. Higher harmonics are rejected by mirrors in front and behind the monochromator. The monochromatic beam leaves the evacuated section 


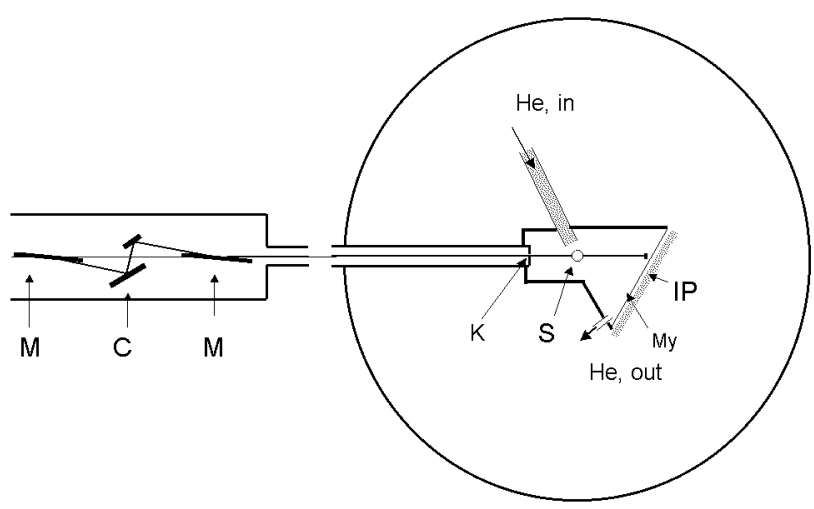

Fig. 2. The beam line ID1 with its ancillary equipment for soft X-ray diffraction. The various parts are not to the same scale. The optical part consists of two mirrors (M) and a double monochromator (C). The monochromatic beam enters into the helium atmosphere through a $15 \mu \mathrm{m}$ Kapton foil $(\mathrm{K})$. The sample crystal (S) is cooled by cold helium gas $(T=40 \mathrm{~K})$. The helium leaving the box is being recovered. The diffracted intensity is recorded on a large image plate (IP) at $23 \mathrm{~cm}$ distance from the sample. The sensitive area of the image plate is $350 \mathrm{~mm} \times 430 \mathrm{~mm}$. There is a $20 \mu \mathrm{m}$ thick Mylar window (polyethylene terephthalate) in front of the image plate.

through a thin Kapton window of $12 \mu \mathrm{m}$ thickness. There are no other windows further upstream.

The monochromatic X-ray beam enters into a helium atmosphere and is diffracted by the protein crystal. The sample, a lysozyme crystal (tetragonal form, see below) with a diameter of about $200 \mu \mathrm{m}$, is opaque for soft X-rays. It is therefore quite easy to see the shadow of the crystal by direct observation of the primary beam of $0.5 \mathrm{~mm}$ diameter.

The diffracted intensity is recorded on a large image plate $(350 \mathrm{~mm} \times 430 \mathrm{~mm}$, pixel size $200 \mu \mathrm{m}$ ) at $230 \mathrm{~mm}$ distance from the sample. The plate is inclined by $30^{\circ}$ in order to cover scattering angles between $-15^{\circ}$ and $+75^{\circ}$ in the vertical direction (Fig. 2). There is a thin window ( $20 \mu \mathrm{m}$ Mylar) in front of the image plate. The image plate is read out off-line. The readout time of 12 minutes was considerably longer than the exposure time of typically some tens of seconds.

\section{Results}

X-ray diffraction from a crystal of tetragonal lysozyme was measured at the following wavelengths: $\lambda_{1}=2.7 \AA, \lambda_{2}=3.5 \AA, \lambda_{3}=4.4 \AA, \lambda_{4}=5.0 \AA$, and $\lambda_{5}=5.7 \AA$. At each wavelength the crystal was rocked through 4 slices of $2^{\circ}$. Diffraction spots were observed at all wavelengths over the total area of the image plate (Figs. $3-7$ ). Good quality diffraction data were obtained even at $\lambda_{5}=5.7 \AA$ where the penetration depth of X-rays decreases to less than $20 \mu \mathrm{m}$. The washing 


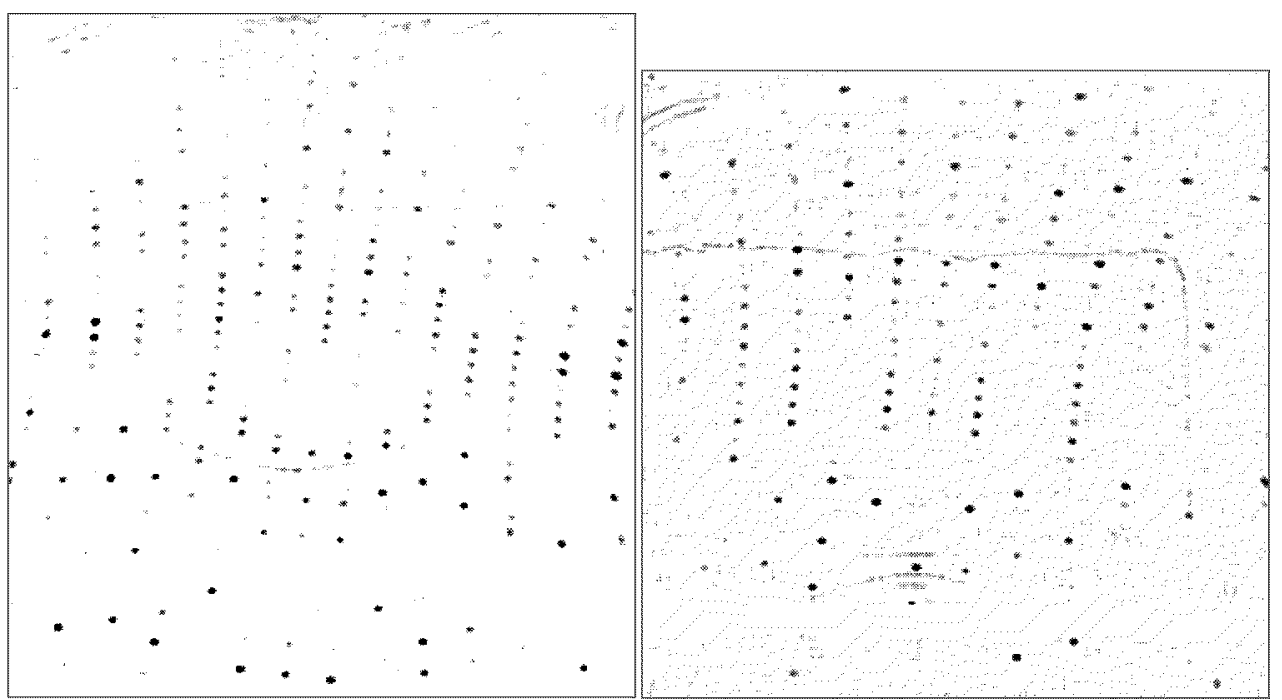

Fig. 3

Fig. 4

Fig. 3. Diffraction of $2.7 \AA$ photons from tetragonal lysozyme. The picture shows the data from a $2^{\circ}$ rotation of the protein crystal. Scattering angles extend from $-15^{\circ}$ (at the bottom of the figure) to $+75^{\circ}$ (at the top of the figure). The background has been subtracted (see text). This wavelength is now being used routinely for protein crystallography at some places [1].

Fig. 4. Diffraction of $3.5 \AA$ photons from lysozyme (conditions as in Fig. 3). This wavelength is of interest for the use of the large anomalous scattering from uranium at its $M_{\mathrm{V}}$ absorption edge.

procedure with oil just before freezing the crystal apparently led to the formation of a thin oil layer which is quite transparent for soft X-rays. Note that in this case the diffraction peaks at larger angles are at least as intense as those observed at smaller angles.

Inspection of Figs. 3 to 7 shows two features:

(1) The spacing between reflections increases with the wavelength. The number of peaks decreases with the inverse cube of the wavelength, which is quite impressive. For wavelengths up to $4.4 \AA$ all peaks predicted by MOSFLM program were found. For still larger wavelengths a similar result was obtained by a special program for indexing written by one of us (H.S.).

(2) The intensity of the Bragg peaks is getting weaker, although the exposure time had been increased from $t=20 \mathrm{~s}$ at $\lambda_{1}$ to $t=120 \mathrm{~s}$ at $\lambda_{5}$.

The second point merits more attention as it provides a measure of the quality of soft X-ray diffraction data. The integrated peak intensities divided by the measuring time decrease with larger wavelengths (Fig. 8). The drastic drop of the intensity at $\lambda=5.7 \AA$ is due to the obligatory change from the undulator 


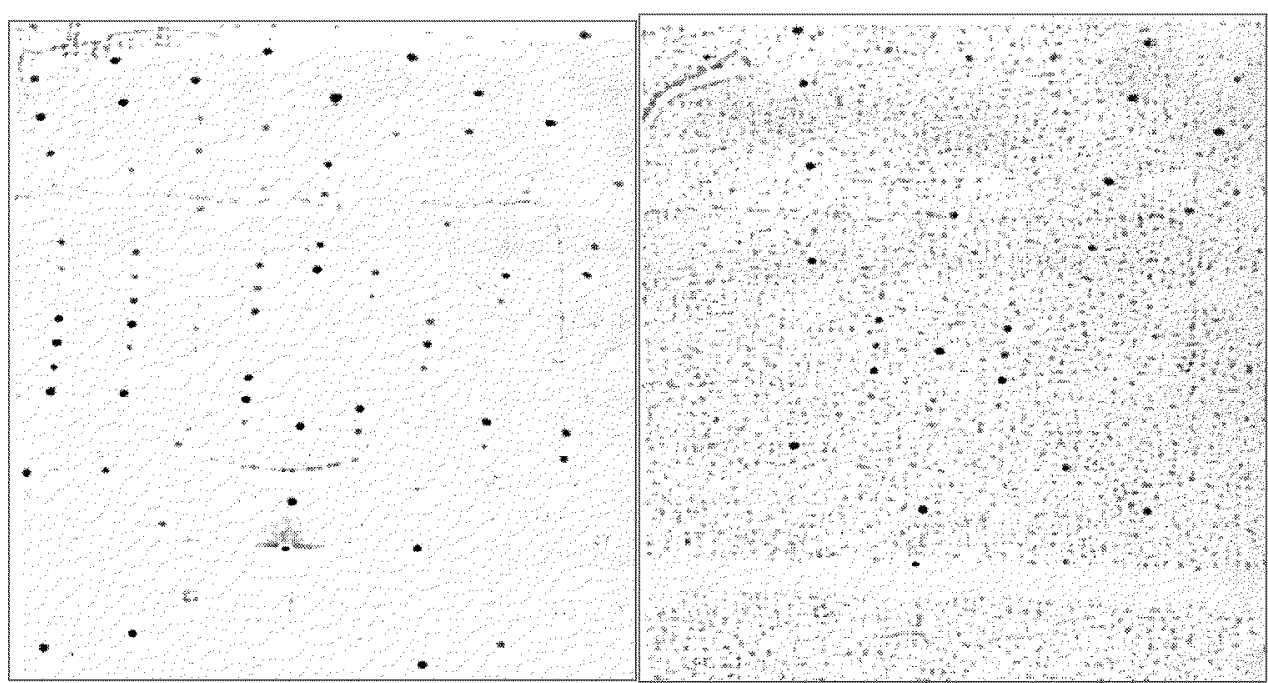

Fig. 5

Fig. 6

Fig. 5. Diffraction of $4.4 \AA$ photons from lysozyme (conditions as in Fig. 3 ). This is the wavelength of the $K$ absorption edge of chlorine.

Fig. 6. Diffraction of $5.0 \AA$ photons from lysozyme (conditions as in Fig. 3). This is the wavelength of the $K$ absorption edge of sulphur.

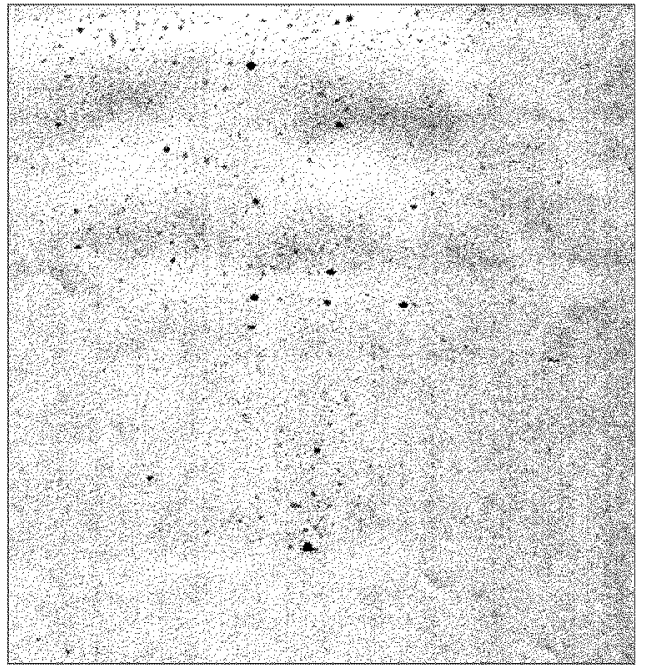

Fig. 7. Diffraction of $5.7 \AA$ photons from lysozyme (conditions as in Fig. 3). This is the wavelength of the $K$ absorption edge of phosphorus.

radiation to the radiation from the wiggler. One reason for the decrease in the measured diffraction intensity is the absorption by windows. Their transmission 


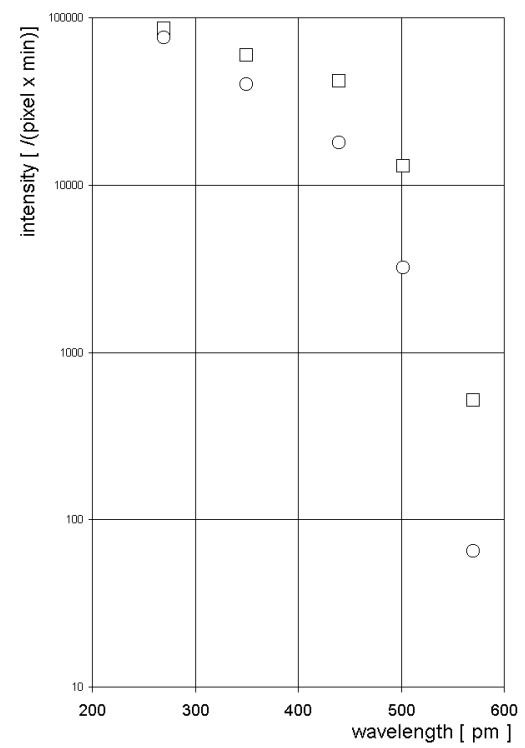

Fig. 8. Integrated intensities of diffraction peaks versus the wavelength (see text). The intensities are given in events per minute and pixel. Only those pixels are counted which are part of the Bragg peak (circles). The squares correspond to intensities after division by the transmission of the windows at the entrance of the helium box ( $15 \mu \mathrm{m}$ Kapton) and in front of the image plate (20 $\mu \mathrm{m}$ Mylar).

as a function of the wavelength is known. Figure 8 shows the integrated intensities after correction for transmission versus the wavelength. These values reflect the influence of three factors: the number of photons hitting the lysozyme crystal, the diffraction power of the crystal and the sensitivity of the image plate. Between $2.7 \AA$ and $5.0 \AA$ this product varies by not more than a factor of 5 .

The diffraction images shown in Figs. 3 to 7 have been filtered using a mask which is comparable to the peak size. In this way the diffraction peaks show up more clearly, while features different from the size of the mask will be reduced. The background scattering, which had been visible before the filter was applied, was mainly due to the scattering of the primary beam by the Kapton foil. At larger angles $\left(2 \theta>50^{\circ}\right)$ the scattering from the Kapton foil is absorbed by the inner surface of the helium box. The boundary line of the region of increased background can be seen most easily in Fig. 4. Any additional window near the sample would lead to a further increase in the background. The open stream cooling system turns out to be the best way to ensure a low background scattering. Moreover, the liquid helium consumption of the cryogenic system from EMBL with about 1 litre/hour is moderate. Recovery of the helium gas is foreseen.

All diffraction images shown in Figs. 3 to 7 exhibit additional structures at the edges, particularly at the side of larger diffraction angles, which are due 
to frost on the cold surface of the plastic window. There was no ice formation inside the box.

From a later experiment it became evident that the exclusion of air inside the cold box is essential for the proper performance of the setup. In that experiment the image plate was introduced into the helium box through a narrow slit. No window in front of the image plate was needed. Although this design is attractive, its drawback was that air entered into the box at each change of the image plate allowing a partial exchange of the helium atmosphere inside the box by air at each change of the image plate. Although the air could be replaced rather quickly by helium, the moisture coming along with the air was deposited as frost near the sample crystal, giving rise to continuously growing ice powder diffraction rings.

\section{Conclusion}

With the introduction of a cold helium atmosphere as sample environment and image plates as detector, the conditions for soft X-ray diffraction have been considerably improved. The helium atmosphere is transparent enough to soft $\mathrm{X}$-rays. The number of windows has been reduced to two, one window separating the helium atmosphere from the vacuum section of the beam line and another one in front of the image plate. The latter one could be avoided as well, once an appropriate mechanism for the reversible introduction of the image plate into the helium atmosphere has been built.

The simultaneous measurement of the diffracted intensity covering a large solid angle remains a technical challenge. Image plates in cylindrically bent form in this respect, appear to be the most suitable detectors. Moreover, their efficiency for soft X-ray with the use of a thinner protective layer $s$ is satisfactory. Further increase in the sensitivity of image plates can be expected.

The off-line read-out time of the image plate of 10 minutes and the additional time for image plate transfer is the rate limiting step in data collection. An on-line scanner for cylindrically bent image plates would ensure a more efficient use of the synchrotron radiation beam time.

\section{Acknowledgment}

The experiments were carried out at the European Synchrotron Radiation Facility (ESRF) at Grenoble. We would like to thank the technical staff of the ID1 beam line of ESRF for their competent support.

\section{References}

[1] M.S. Weiss, T. Sicker, K. Djinovic-Carugo, R. Hilgenfeld, Acta Crystallogr. D 57, $689(2001)$. 
[2] W.A. Hendrickson, M.M. Teeter, Nature 290, 107 (1981).

[3] B.-C. Wang, Methods Enzymol. 115, 90 (1985).

[4] Z. Dauter, M. Dauter, E. de la Fortelle, G. Bricogne, G.M. Sheldrick, J. Mol. Biol. 289, 83 (1999).

[5] M.S. Weiss, T. Sicker, R. Hilgenfeld, Structure 9, 771 (2001).

[6] W.A. Hendrickson, C.M. Ogata, Methods Enzymol. 276, 494 (1997).

[7] S. Stuhrmann, R. Braunwarth, R. Doose, F. Dauvergne, A. Gabriel, A. Knöchel, M. Marmotti, H.B. Stuhrmann, J. Thomas, C. Trame, M.S. Lehmann, J. Synchrotr. Radiat. 4, 298 (1997).

[8] L.B. Sorensen, J.O. Cross, B. Newville, B. Ravel, J.J. Rehr, H. Stragier, C.E. Bouldin, J.C. Woicik, in: Resonant X-ray Scattering, Theory and Applications, Eds. G. Materlik, C.J. Sparks, K. Fischer, North-Holland, Amsterdam 1994, p. 389 .

[9] P. Carpentier, M. Capitan, M.-L. Chesne, E. Fanchon, R. Kahn, S. Lequien, H. Stuhrmann, D. Thiaudière, J. Vicat, W. Zajac, P. Zielinski, J. Alloys Comp. 328, 64 (2001).

[10] S. Stuhrmann, M. Hütsch, C. Trame, J. Thomas, H.B. Stuhrmann, J. Synchrotr. Radiat. 2, 83 (1995).

[11] S. Stuhrmann, K.S. Bartels, M. Hütsch, M. Marmotti, Z. Sayers, J. Thomas, C. Trame, H.B. Stuhrmann, in: Structural Studies on Crystals, Nauka, Fizmatlit, Moskva 1996, p. 276 (in Russian).

[12] R. Kahn, P. Carpentier, C. Berthet-Colominas, M. Capitan, M.-L. Chesne, E. Fanchon, S. Lequien, D. Thiaudière, J. Vicat, P. Zielinski, H. Stuhrmann, J. Synchrotr. Radiat. 7, 131 (2000).

[13] P. Carpentier, C. Berthet-Colominas, M. Capitan, M.-L. Chesne, E. Fanchon, S. Lequien, H. Stuhrmann, D. Thiaudière, J. Vicat, P. Zielinski, R. Kahn, Cell. Molec. Biol. 46, 915 (2000). 